\title{
BMJ Open Acupuncture for emotional disorders in patients with migraine: a systematic review protocol
}

\author{
Ning Sun (D), Mingsheng Sun (1) , Zhengjie Li, Rui-Rui Sun, Ling Zhao, Jiao Chen, \\ Fan-rong Liang (D)
}

\begin{abstract}
To cite: Sun N, Sun M, Li Z, et al. Acupuncture for emotional disorders in patients with migraine: a systematic review protocol. BMJ Open 2020;10:e034290. doi:10.1136/ bmjopen-2019-034290

- Prepublication history for this paper is available online. To view these files, please visit the journal online (http://dx.doi. org/10.1136/bmjopen-2019034290).
\end{abstract}

Received 13 September 2019 Revised 18 November 2019 Accepted 28 November 2019
Check for updates

(C) Author(s) (or their employer(s)) 2020. Re-use permitted under CC BY-NC. No commercial re-use. See rights and permissions. Published by BMJ.

Acupuncture and Tuina School/ The 3rd Teaching Hospital, Chengdu University of Traditional Chinese Medicine, Chengdu, China

Correspondence to Dr Fan-rong Liang; acuresearch@126.com

\section{ABSTRACT}

Introduction Migraine is the second-leading cause of years lived with disability worldwide. The high prevalence of migraine-related emotional disorders is often overlooked. Acupuncture is often used to treat both migraine and emotional disorders. This systematic review protocol aims to analyse whether acupuncture is effective for treating emotional disorders in patients with migraine. Methods and analysis Nine databases will be searched from inception to may 2019: cochrane central register of controlled trials, medline, embase, allied and complementary medicine database, cinahl, china national knowledge infrastructure, chinese biomedical literature database, vip database and wanfang database. Randomised controlled trials (rcts) of acupuncture therapy for migraine with emotional functioning outcomes, which were reported in chinese or english, will be included. The primary outcome is the change in emotional functioning. Study selection, data extraction and assessment of the risk of bias will be performed independently by two or more reviewers. Revman software (v.5.3) will be used to perform the assessment of the risk of bias and data synthesis. Ethics and dissemination Ethics approval is not be needed because the data will not contain individual patient data, and there are no concerns about privacy. The results of this meta-analysis will be disseminated through publication in a peer-reviewed journal or relevant conference.

Trial registration number CRD42019139433.

\section{INTRODUCTION}

\section{Description of the condition}

Migraine is a bio-behavioural disorder characterised by a unilateral throbbing or pulsating headache and accompanied by associated symptoms (nausea, photophobia or phonophobia). ${ }^{12}$ The prevalence of $14.9 \%$ has been reported in the usa, ${ }^{3}$ with about $16 \%-18 \%$ of women and $6 \%-8 \%$ of men. ${ }^{4}$ Meanwhile, migraine is the second-leading cause of years lived with disability worldwide. ${ }^{5}$

Often overlooked is the high prevalence of migraine-related emotional disorders, which encompassing a series of disorders with high rates of comorbidity, high levels of negative affect and low levels of positive
Strengths and limitations of this study

This study will be the first ever meta-analysis analysing acupuncture in the treatment of emotional disorders in patients with migraine.

- The systematic review may reveal whether acupuncture plays an anti-anxiety and anti-depression by solving the primary migraine, or whether acupuncture treats the accompanying emotional disorders of migraine alone.

- As different scales have been used in published trials, a pooled analysis of all included studies may not be possible; however, subgroup analyses will be performed according to different outcomes.

- Different types of acupuncture may cause considerable heterogeneity in this review.

affect, ${ }^{67}$ especially depression and anxiety. ${ }^{89}$ Migraineurs are over 2.5 times more likely to suffer from depression compared with nonmigraineurs. ${ }^{10-12}$ Global burden of disease (gbd) 2016 confirmed that severe depression and anxiety were the main reasons for the high disability rate of women. ${ }^{5}$ If left untreated, the comorbid emotional disorders can increase migraine-related disability, reduce the quality of life and negatively impact treatment and clinical outcomes. ${ }^{13-15}$ In present, migraine and emotional disorders are often treated individually. ${ }^{16}$ Pharmacological therapies, such as selective serotonin reuptake inhibitors, serotonin-norepinephrine reuptake inhibitors and tricyclic antidepressants are recommended for depression. However, such treatments are often associated with various side-effects, including headache, nausea and vomiting, and worsened memory. ${ }^{1718}$

\section{Description of the intervention}

Acupuncture treatment, as a part of traditional Chinese medicine, involves insertion and stimulation of fine needles into specific points on the body to facilitate recovery of health. Acupuncture is generally considered safe ${ }^{19}$ and has been shown may have effects on 
migraine and emotions disorders. An individual patient data meta-analysis showed that acupuncture was associated with a statistically significant reduction in symptoms of pain-related depression. ${ }^{20} \mathrm{~A}$ Cochrane systematic review indicated that acupuncture as adjunctive treatment could reduce the frequency of migraine. ${ }^{21}$ But this review did not conduct a quantitative or qualitative analysis on the effect of acupuncture for migraine-related emotional disorders. Furthermore, an increasing number of clinical studies have shown the effect of acupuncture on emotional disorders in migraineurs. ${ }^{22} 23$

\section{How the intervention might work}

It is confirmed by many studies that acupuncture activates the release of some pain suppressors, such as endorphins, in the central nervous system. ${ }^{24}{ }^{25} \mathrm{Li}$ et al have concluded that the impairment of the homoeostasis of the trigeminovascular nociceptive pathway was involved in the neural pathophysiology of migraines, and acupuncture could help to restore this imbalance. ${ }^{26}$ Yue et al have shown that electro-acupuncture exhibited the antidepressant effect and alleviated the hippocampal neuroinflammation. ${ }^{27}$ Meanwhile, the stimulation of specific acupoint by electro-acupuncture could cause relaxation, calmness and reduce the feeling of tension or distress. ${ }^{28}$ Furthermore, Shao et al found that strong manual acupuncture stimulation could reduce pain-induced anxiety and phosphorylation of extracellular signal-regulated kinase in the anterior cingulate cortex (ACC) ${ }^{29} \mathrm{Du}$ et al confirmed that electro-acupuncture could decrease pain-induced anxiety-like behaviours by suppressing protein kinase mzeta activity in the ACC. ${ }^{30}$ Meanwhile, it has been shown that there is a shared neurobiological mechanism for emotional disorders and migraine. ${ }^{31-33}$ In addition, it was observed that reduced migraine days were accompanied by decreased anxiety. ${ }^{34}$

Whether acupuncture plays an anti-anxiety and antidepression by solving the primary migraine, or whether acupuncture treats the accompanying emotional disorders of migraine alone? This point needs to be further studied based on clarifying whether acupuncture is effective for the emotional disorders of migraine.

\section{Why it is important to perform this review}

Given the potential associations among emotional disorders and increased risk of disease progression and migraine-related burden, it is important to understand the impact of migraine treatment on the management of these comorbidities. In this circumstances, comorbid emotional disorders need to be considered when devising a treatment plan for the patient with migraine. ${ }^{35} 36$ However, there is no systematic reviews to evaluate the efficacy of acupuncture in the treatment of migraine accompanied with emotional disorders. Therefore, a comprehensive review of acupuncture on emotional disorders in patients with migraine will provide a useful estimate of overall effects of complex interventions, an estimate of most interest to patients, practitioners and providers.

\section{Objectives}

The systematic review aims to obtain a relatively convincing conclusion of whether acupuncture is effective for treating emotional disorders in patients with migraine.

\section{METHODS \\ Criteria for including studies in this review Types of studies}

RCTs of acupuncture therapy for migraine with emotional functioning outcomes, which were reported in Chinese or English, will be included. Quasi-RCTs and uncontrolled clinical trials will be excluded.

\section{Types of participants}

Patients were diagnosed either with acute or chronic episodes of migraine, will be included regardless of the age, gender and source of cases. The diagnosis will be based on the International Classification of Headache Disorders. ${ }^{37}$

\section{Types of interventions}

The style of acupuncture considered in the studies is defined as needle stimulation of acupoints, including body acupuncture (manual/electro), auricular acupuncture and scalp acupuncture.

\section{Types of comparator(s)/control}

We included and classified the comparators in studies as follows:

1. Acupuncture versus invasive sham $/$ minimal acupuncture.

2. Acupuncture versus non-invasive placebo acupuncture.

3. Acupuncture versus waiting list/no treatment/usual care.

Studies comparing different acupoints or different forms of acupuncture will be excluded.

Types of outcome measures

\section{Primary outcomes}

Emotional functioning outcomes scale: the MigraineSpecific Quality-of-Life Questionnaire (MSQ) emotional functional subscale, Allgemeine Depressions Skala, Hamilton Depression Scale and so on.

\section{Secondary outcomes}

1. The change in the frequency of migraine attacks.

2. Days of migraine (days/month).

3. Visual analogue scale.

4. MSQ.

5. Adverse events, including the number of participants dropped out and the number of participants reported adverse events. 


\section{Search methods for identification of studies}

\section{Electronics searches}

The following databases will be searched from inception to December 2019: Cochrane Central Register of Controlled Trials, MEDLINE, EMBASE, Allied and Complementary Medicine Database, CINAHL, China National Knowledge Infrastructure, Chinese Biomedical Literature Database, VIP Database and Wanfang Database.

The following terms will be searched: headache, migraine, cephalgia, cephalalgia, acupuncture, manual acupuncture, electro-acupuncture and auricular acupuncture. The search strategy for MEDLINE is shown in table 1 . In the Chinese databases, the equivalent search words will be used.

\section{Searching other resources}

Clinical trial registries, like the WHO International Clinical Trial Registry Platform, Chinese clinical registry and ClinicalTrials.gov, will be searched for ongoing trials with unpublished data. The reference lists of all potential publications, including relevant systematic reviews, will be manually retrieved and reviewed to further locate additional trials. Incomplete data will be obtained by contacting the corresponding authors.

\section{Data collection and analysis}

\section{Selection of studies}

The titles and abstracts of all searched studies will be screened independently by two reviewers (NS and MS) according to the inclusion criteria. The full text of eligible studies will be reviewed if necessary. Any disagreements will be arbitrated by a third reviewer (ZL). Excluded studies will be listed in a table with reasons for their exclusion. The study selection procedure is shown in a Preferred Reporting Items for Systematic Reviews and Meta-Analyses flow chart (figure 1).

\section{Data extraction and management}

The following information will be extracted from all eligible studies by two independent reviewers (NS and MS) and entered a predefined data acquisition form: reference ID, author information, publication year, participant characteristics, acupuncture intervention, control intervention, outcomes at all reported time points, and adverse effects, medication intake, duration of follow-up and so on. Details about acupuncture techniques will be extracted according to Standards for Reporting Interventions in Clinical Trials of Acupuncture.$^{38}$ Information not available from the studies will be obtained by contacting the corresponding authors. Any disagreements will be arbitrated by a third reviewer (R-RS). Cross-check of all data will be done by NS and MS and transferred into RevMan software (V.5.3).

\section{Assessment of risk of bias}

The risk of bias for each study will be evaluated by two or more independent reviewers (NS and M-SS) using the Cochrane Handbook for Systematic Reviews of Interventions. ${ }^{39}$ The following six domains will be assessed:

\begin{tabular}{|c|c|}
\hline no. & search items \\
\hline$\# 1$ & randomized controlled trial [pt] \\
\hline \#2 & controlled clinical trial [pt] \\
\hline$\# 3$ & randomized [tiab] \\
\hline$\# 4$ & placebo [tiab] \\
\hline \#5 & clinical trials [mesh] \\
\hline \#6 & randomly [tiab] \\
\hline$\# 7$ & trial [ti] \\
\hline \#8 & $\# 1$ or \#2 or \#3 or \#4 or \#5 or \#6 or \#7 \\
\hline \#9 & humans [mesh] \\
\hline$\# 10$ & $\# 8$ and \#9 \\
\hline$\# 11$ & headache disorders [mesh] \\
\hline$\# 12$ & headache [mesh] \\
\hline$\# 13$ & $\begin{array}{l}\text { (headache or migraine or cephalgia or cephalalgia } \\
\text { or chronic migraine): } \mathrm{ti}, \mathrm{ab}\end{array}$ \\
\hline$\# 14$ & $\# 11$ or \#12 or \#13 \\
\hline \#15 & acupuncture therapy [mesh] \\
\hline$\# 16$ & $\begin{array}{l}\text { (acupuncture or body acupuncture or manual } \\
\text { acupuncture or electroacupuncture or electro- } \\
\text { acupuncture or auricular acupuncture or laser } \\
\text { acupuncture or warm needling): ti,ab }\end{array}$ \\
\hline \#17 & \#15 or \#16 \\
\hline \#18 & $\# 10$ and $\# 14$ and $\# 17$ \\
\hline
\end{tabular}

selection bias, performance bias, detection bias, attrition bias, reporting bias and other sources of bias. Trials will be evaluated and classified into three levels: low risk, high risk and unclear. Corresponding authors will be contacting fir unclear items. Any disagreements will be arbitrated by a third reviewer (R-RS).

\section{Measures of treatment effect}

Efficacy data will be synthesised and statistically analysed in RevMan V.5.3. For dichotomous data, a risk ratio with 95\% CIs will be used for analysis. For continuous data, a mean difference or a standard mean difference (SMD) with $95 \%$ CIs will be used for analysis. SMD will be used if different assessment tools were used.

\section{Unit of analysis issues}

Due to the lack of individual patient data, an analysis will be based on aggregated outcome data.

\section{Dealing with missing data}

The corresponding authors or relevant authors will be contacted by reviewers (NS and MS) to obtain missing data. An intention-to-treat analysis will be performed in the absence of missing data. ${ }^{40}$ If possible, a sensitivity analysis will be performed to address the potential impact of missing data. ${ }^{41}$ The potential impact of missing data will be addressed in the discussion if necessary. 


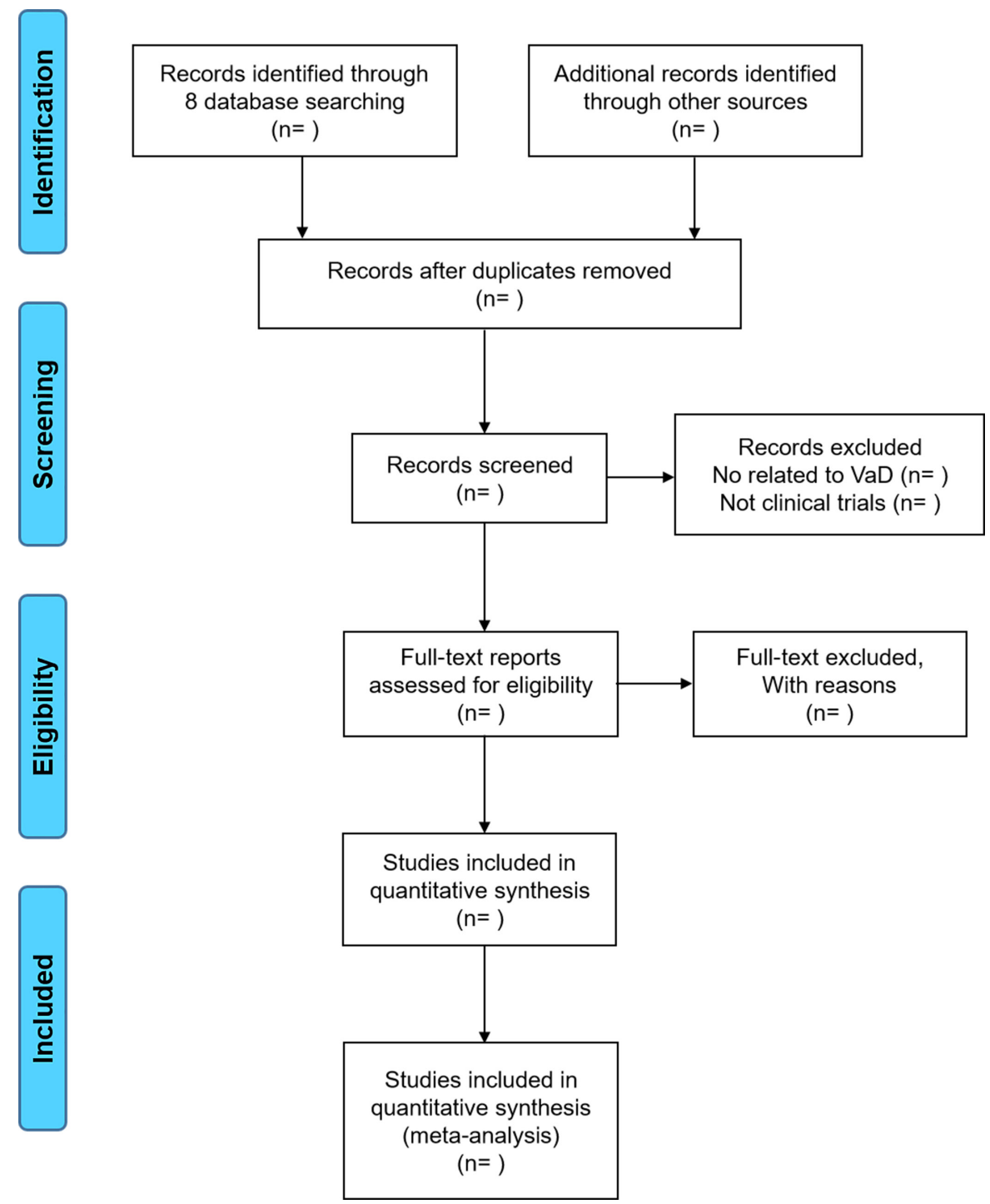

Figure 1 Flow diagram of the study selection process.

\section{Assessment of heterogeneity}

$\chi^{2}$ test with a significance level of $p<0.1$ will be used in the forest plot for investigating statistical heterogeneity. Moreover, an $\mathrm{I}^{2}$ test will be calculated for quantifying inconsistency. If the heterogeneity tests show little or no statistical heterogeneity in these trials $\left(\mathrm{I}^{2}<50 \%\right)$, the fixed effects model will be used for the pooled data. The random-effects model will be used with heterogeneous data $\left(50 \%<\mathrm{I}^{2}<75 \%\right)$. If there is considerable heterogeneity, meta-analysis will not be performed.

\section{Assessment of reporting biases}

Funnel plots will be generated to observe the potential reporting biases when more than 10 studies are included.

\section{Data synthesis}

Data synthesis will be performed with clinical data, using RevMan software (V.5.3). The fixed-effects model will be used for the pooled data if the statistical heterogeneity is detected as little or low. Otherwise, the random-effects model will be used. If necessary, subgroup analysis will be performed, or the potential reasons will be analysed.

Subgroup analysis and investigation of heterogeneity

If data are available, subgroup analysis will be performed. Variations in the characteristics like acupuncture types, control intervention types and short/long term effects will be considered. And subgroup will be conducted to interpret the heterogeneity.

Sensitivity analysis

A sensitivity analysis will be conducted to monitor the robustness of the primary decision made in the review process, if possible. Several decision nodes, such as methodological weaknesses, sample size and missing data, will be considered.

\section{Summary of evidence}

Two reviewers will assess independently the quality of evidence for main outcomes using the grading of recommendations assessment, development, and evaluation 
system approach, ${ }^{42}$ and present the result in 'summary of findings' tables in the final report. The assessments of evidence quality will be rated 'high', 'moderate', 'low' or 'very low' ${ }^{43}$ the quality of evidence of a specific study will be assessed according to the risk of bias, imprecision, inconsistency, indirectness, publication bias, effect size or doseresponse relation.

\section{Patient and public involvement \\ No patient involved.}

\section{Ethics and dissemination}

The results of this meta-analysis will be disseminated through publication in a peer-reviewed journal or relevant conference.

\section{DISCUSSION}

This meta-analysis will provide a relatively convincing conclusion of whether acupuncture is effective for treating emotional disorders in patients with migraine. Conclusions drawn from this review may benefit migraineurs, clinicians and policy makers. The process of conducting this review will be divided into four parts: identification, study inclusion, data extraction and data synthesis. If amendments to this protocol are necessary, we will provide the date of each amendment with statement of the changes and the corresponding reasons.

Contributors NS and F-rL designed the systematic review. NS drafted the protocol. ZL, LZ and MS revised the manuscript. JC, R-RS and NS participated in the design of data synthesis and analysis plan. All authors have read and approved the publication of the protocol.

Funding This work was funded by the National Natural Science Foundation of China (grant number 81590951).

Competing interests None declared.

Patient consent for publication Not required.

Ethics approval Ethics approval is not be needed because the data will not contain individual patient data, and there are no concerns about privacy.

Provenance and peer review Not commissioned; externally peer reviewed.

Open access This is an open access article distributed in accordance with the Creative Commons Attribution Non Commercial (CC BY-NC 4.0) license, which permits others to distribute, remix, adapt, build upon this work non-commercially, and license their derivative works on different terms, provided the original work is properly cited, appropriate credit is given, any changes made indicated, and the use is non-commercial. See: http://creativecommons.org/licenses/by-nc/4.0/.

\section{ORCID iDs}

Ning Sun http://orcid.org/0000-0002-1064-5705

Mingsheng Sun http://orcid.org/0000-0002-6748-5087

Fan-rong Liang http://orcid.org/0000-0001-8518-9268

\section{REFERENCES}

1 Stovner LJ, Nichols E, Steiner TJ, et al. Global, regional, and national burden of migraine and tension-type headache, 1990-2016: a systematic analysis for the global burden of disease study 2016 . Lancet Neurol 2018;17:954-76.

2 Goadsby P. Incredible progress in migraine for an era of better care. Nat Rev Neurol 2015;11:621-2.

3 Burch RC, Loder S, Loder E, et al. The prevalence and burden of migraine and severe headache in the United States: updated statistics from government health surveillance studies. Headache 2015;55:21-34.

4 Fumal A, Schoenen J. Current migraine management - patient acceptability and future approaches. Neuropsychiatr Dis Treat 2008;4:1043-57.

5 Vos T, Abajobir AA, Abate KH, et al. Global, regional, and national incidence, prevalence, and years lived with disability for 328 diseases and injuries for 195 countries, 1990-2016: a systematic analysis for the global burden of disease study 2016. Lancet 2017;390:1211-59.

6 Barlow DH, Farchione TJ, Bullis JR, et al. The unified protocol for Transdiagnostic treatment of emotional disorders compared with Diagnosis-Specific protocols for anxiety disorders: a randomized clinical trial. JAMA Psychiatry 2017;74:875-84.

7 Mohajerin B, Bakhtiyar M, Olesnycky OS, et al. Application of a transdiagnostic treatment for emotional disorders to body dysmorphic disorder: a randomized controlled trial. J Affect Disord 2019;245:637-44.

8 Breslau N, Davis GC, Migraine DGC. Migraine, physical health and psychiatric disorder: a prospective epidemiologic study in young adults. J Psychiatr Res 1993;27:211-21.

9 Lantéri-Minet M, Radat F, Chautard $\mathrm{M}-\mathrm{H}$, et al. Anxiety and depression associated with migraine: influence on migraine subjects disability and quality of life, and acute migraine management. Pain 2005;118:319-26.

10 Lipton RB, Hamelsky SW, Kolodner KB, et al. Migraine, quality of life, and depression: a population-based case-control study. Neurology 2000;55:629-35.

11 Breslau N, Schultz LR, Stewart WF, et al. Headache and major depression: is the association specific to migraine? Neurology 2000;54:308.

12 Zwart J-A, Dyb G, Hagen K, et al. Depression and anxiety disorders associated with headache frequency. The Nord-Trøndelag health study. Eur J Neurol 2003;10:147-52.

13 Bair MJ, Robinson RL, Eckert GJ, et al. Impact of pain on depression treatment response in primary care. Psychosom Med 2004;66:17-22.

14 Goh KK, Chen C-H, Chiu Y-H, et al. Lamotrigine augmentation in treatment-resistant unipolar depression: a comprehensive metaanalysis of efficacy and safety. J Psychopharmacol 2019;33:700-13.

15 Rosenzweig-Lipson S, Beyer CE, Hughes ZA, et al. Differentiating antidepressants of the future: efficacy and safety. Pharmacol Ther 2007:113:134-53.

16 Argoff CE. The coexistence of neuropathic pain, sleep, and psychiatric disorders: a novel treatment approach. Clin J Pain 2007;23:15-22.

17 Asmundson GJG, Katz J. Understanding the co-occurrence of anxiety disorders and chronic pain: state-of-the-art. Depress Anxiety 2009;26:888-901.

18 Louter MA, Pijpers JA, Wardenaar KJ, et al. Symptom dimensions of affective disorders in migraine patients. $J$ Psychosom Res 2015;79:458-63.

19 Vincent C. The safety of acupuncture: acupuncture is safe in the hands of competent practitioners. BMJ 2001;323:467-8.

20 MacPherson $\mathrm{H}$, Vickers $\mathrm{A}$, Bland $\mathrm{M}$, et al. Acupuncture for chronic pain and depression in primary care: a programme of research. Programme Grants Appl Res 2017;5:1-316.

21 Linde K, Allais G, Brinkhaus B, et al. Acupuncture for the prevention of episodic migraine. Cochrane Database Syst Rev 2016:CD001218.

22 Zhao L, Chen J, Li Y, et al. The long-term effect of acupuncture for migraine prophylaxis: a randomized clinical trial. JAMA Intern Med 2017;177:508-15.

23 Wang L-P, Zhang X-Z, Guo J, et al. Efficacy of acupuncture for migraine prophylaxis: a single-blinded, double-dummy, randomized controlled trial. Pain 2011;152:1864-71.

24 Han J-S. Acupuncture and endorphins. Neurosci Lett 2004;361:258-61.

25 Goldman N, Chen M, Fujita T, et al. Adenosine A1 receptors mediate local anti-nociceptive effects of acupuncture. Nat Neurosci 2010;13:883-8.

26 Li Z, Zeng F, Yin T, et al. Acupuncture modulates the abnormal brainstem activity in migraine without aura patients. Neuroimage Clin 2017;15:367-75

27 Yue N, Li B, Yang L, et al. Electro-Acupuncture alleviates chronic unpredictable stress-induced Depressive- and anxiety-like behavior and hippocampal neuroinflammation in rat model of depression. Front Mol Neurosci 2018;11:149.

28 Hsu C-C, Weng C-S, Liu T-S, et al. Effects of electrical acupuncture on Acupoint BL15 evaluated in terms of heart rate variability, pulse rate variability and skin conductance response. Am J Chin Med 2006;34:23-36.

29 Shao X-M, Shen Z, Sun J, et al. Strong Manual Acupuncture Stimulation of "Huantiao" (GB 30) Reduces Pain-Induced Anxiety and 
$\mathrm{p}$-ERK in the Anterior Cingulate Cortex in a Rat Model of Neuropathic Pain. Evid Based Complement Alternat Med 2015;2015:235491.

30 Du J, Fang J, Wen C, et al. The effect of electroacupuncture on PKMzeta in the ACC in regulating anxiety-like behaviors in rats experiencing chronic inflammatory pain. Neural Plast 2017;2017:3728752.

31 Pesa J, Lage MJ. The medical costs of migraine and comorbid anxiety and depression. Headache 2004;44:562-70.

32 Schwedt TJ, Chong CD, Chiang C-C, et al. Enhanced pain-induced activity of pain-processing regions in a case-control study of episodic migraine. Cephalalgia 2014;34:947-58.

33 Russo A, Tessitore A, Esposito F, et al. Pain processing in patients with migraine: an event-related fMRI study during trigeminal nociceptive stimulation. J Neurol 2012;259:1903-12.

34 Liu J, Mu J, Liu Q, et al. Brain structural properties predict psychologically mediated hypoalgesia in an 8-week sham acupuncture treatment for migraine. Hum Brain Mapp 2017;38:4386-97.

35 Breslau N, Merikangas K, Bowden CL. Comorbidity of migraine and major affective disorders. Neurology 1994;44:S17-22.

36 Merikangas KR, Stevens DE. Comorbidity of migraine and psychiatric disorders. Neurol Clin 1997;15:115-23.
37 Headache Classification Subcommittee of the International Headache Society. The International classification of headache disorders: 2nd edition. Cephalalgia 2004;24(Suppl 1):9-160.

38 MacPherson H, Altman DG, Hammerschlag R, et al. Revised standards for reporting interventions in clinical trials of acupuncture (stricta): extending the CONSORT statement. J Evid Based Med 2010;3:140-55.

39 Wang S, Yang H, Zhang J, et al. Efficacy and safety assessment of acupuncture and nimodipine to treat mild cognitive impairment after cerebral infarction: a randomized controlled trial. BMC Complement Altern Med 2016;16:361.

40 Abraha I, Cozzolino F, Orso M, et al. A systematic review found that deviations from intention-to-treat are common in randomized trials and systematic reviews. J Clin Epidemiol 2017;84:37-46.

41 Porta N, Bonet C, Cobo E. Discordance between reported intentionto-treat and per protocol analyses. J Clin Epidemiol 2007;60:663-9.

42 Guyatt GH, Oxman AD, Vist GE, et al. Grade: an emerging consensus on rating quality of evidence and strength of recommendations. BMJ 2008;336:924-6.

43 Guyatt GH, Oxman AD, Schünemann HJ, et al. Grade guidelines: a new series of articles in the Journal of clinical epidemiology. J Clin Epidemiol 2011;64:380-2. 